\title{
Granulomatosis con poliangeitis: Manifestaciones clínicas en cabeza y cuello.
}

\section{Granulomatosis with polyangiitis: clinical manifestations in the head and neck.}

Pág. 2,11

Recibido: $26-03-2020$

Aceptado: 08-06-2020

Dr. Josué D. Ramírez Esquivel ${ }^{1}$

1. Médico General,Trabajador independiente, Costa Rica.

\section{RESUMEN}

La granulomatosis con poliangeitis es una enfermedad poco común, cuya incidencia anual es de 8-10 casos por millón de personas, causada por una reacción autoinmune inusual asociada a la elevación de los anticuerpos anticitoplasma de neutrófilos (ANCA) que ocasionan vasculitis de pequeños vasos e inflamación granulomatosa. Estas reacciones ocasionan síntomas que usualmente comienzan de manera localizada, afectando la cabeza y el cuello, que progresan a una afectación sistémica con pobre pronóstico, sin embargo en ocasiones estos pueden ser pasados por altos y atrasar su diagnóstico. El artículo se centra en mencionar las manifestaciones clínicas más importantes en la cabeza y el cuello. El diagnóstico es difícil y se basa en las manifestaciones clínicas, asociadas a un análisis histopatológico y positividad de Ios ANCA. El tratamiento temprano ayuda a mejorar el pronóstico de esta, y se necesita de un equipo multidisciplinario para el diagnóstico y tratamiento de esta enfermedad, para así lograr una remisión y prevenir la mayor cantidad de efectos adversos de los tratamientos.

\section{ABSTRACT}

Granulomatosis with polyangiitis is a rare disease, with an anual incidence of 8-10 cases per million people, it's caused by an unusual autoimmune reaction associated with antineutrophil cytoplasmic antibodies (ANCA), producing small-vessel vasculitis and granulomatous inflammation. These reactions result in signs and symptoms, usually localized starting in the head and neck region, progressing to a systemic form with a poor prognosis, however, some of these symptoms can be overlooked and delay the diagnosis. This article centers in mentioning the most important clinical manifestations in the head and neck. Diagnosis is difficult, and it's based on clinical manifestations associated with 
histopathological analysis and ANCA positivity. Early treatment helps improving the prognosis, and a multi-disciplinary cooperation is needed for the diagnosis and treatment of this disease, to adequately achieve the remission and to prevent most of the adverse effects of the treatments.

\section{PALABRAS CLAVE}

Granulomatosis con poliangeitis, Wegener, otorrinolaringología, ANCA, vasculitis, Reumatología.

\section{KEY WORDS}

Granulomatosis with polyangiitis, Wegener, otolaryngology, ANCA, vasculitis, rheumatology.

\section{INTRODUCCIÓN}

Las vasculitis sistémicas son enfermedades autoinmunes poco comunes y de etiología no del todo esclarecida, de manera general se van a dividir en subgrupos dependiendo del tamaño el vaso que estén afectando, la granulomatosis con poliangeitis (GPA) previamente llamada de Wegener se caracteriza por la vasculitis de vasos pequeños e inflamación granulomatosa necrotizante con una deficiencia de depósito de inmuncomplejos o complemento (pauciinmune).

Esto coincide con un aumento en los títulos de los anticuerpos anticitoplasma de neutrófilos contra la proteinasa 3 (PR3-ANCA), la GPA según su espectro clínico se divide en localizada y sistémica (1) (2).

\section{EPIDEMIOLOGÍA}

Si bien esta enfermedad se considera rara su incidencia anual ronda los 8 a 10 casos por millón de habitantes, puede afectar a cualquier grupo etario, sin embargo tiene un pico entre los 64-75 años y una incidencia en la edad pediátrica de 0.3 por millón (3). Esta afecta a todos los grupos étnicos y raciales, pero, destaca una clara predominancia en pacientes caucásicos, y en comparación con los hispanos, estos últimos tienden a presentar la enfermedad de manera más severa $(3,4)$. Se pueden encontrar diferencias geográficas, donde países del norte de Europa como Reino Unido y Alemania cuentan con mayor incidencia de GPA en comparación con el sur como España, y otros sitios como Japón donde el espectro de las vasculitis relacionadas a ANCA tiende a ser más asociado a la poliangeitis microscópica que a GPA $(3,4,5)$

\section{PATOGÉNESIS}

El mecanismo fisiopatológico más estudiado en la GPA está asociado con la autoinmunidad del PR3-ANCA, sin embargo la génesis de estos autoanticuerpos es desconocido, y en esta intervienen factores genéticos, ambientales y biológicos (1). Este anticuerpo al estimular el neutrófilo ocasiona la liberación de factores proinflamatorios y de adhesión celular (6).

Dichas respuestas conducen a la degranulación de enzimas y radicales de oxigeno tóxicos, además del reclutamiento de otras células de la inflamación que perpetuarán y aumentarán la reacción, que resulta en daño endotelial y en aumento de la expresión de antígenos sobre la superficie de los neutrófilos, incrementando así la reactividad de los ANCA sobre ellos $(1,6)$.

El origen de esta respuesta autoinmune puede hallarse correlacionado al mimetismo molecular, se ha encontrado evidencia de autoantígenos creados a partir de péptidos bacterianos de 
S. aureus y otras bacterias a partir de sus fimbrias como E. coli y K. pneumoniae, que están asociadas a otros tipos de ANCA, como la dirigida contra la proteína de membrana lisosomal 2 (LAMP-2). Esta última, en modelos animales, se ha encontrado podría causar consecuencias similares a las de la GPA en el ser humano, y también ha podido ser detectada en seres humanos con GPA y por lo tanto se cree está involucrada en la génesis de esta respuesta inmune desordenada (6).

La comprensión de estos mecanismos es muy importante para llegar a obtener la mejor conducta terapéutica, ya que, sin tratamiento el curso natural de la enfermedad es rápidamente escalonado y fatal, lo cual justifica un tratamiento agresivo (7).

\section{MANIFESTACIONES CLÍNICAS}

La GPA se subdivide en localizada si esta afectan vía respiratoria superior o inferior, o sistémica si afecta a riñón o pulmón. Las manifestaciones sistémicas adquieren real importancia e inclusive llegan a causar lesiones potencialmente mortales $(6,8)$. En el curso natural de la GPA lo más común es que al momento del diagnóstico los síntomas sean localizados, y conforme avanza comienza a presentar síntomas sistémicos, razón por la cual su pronto diagnóstico y tratamiento conlleva a una importante disminución de la morbimortalidad $(2,9,10)$.

En un estudio cohorte por Abdou et al, se estudiaron un total de 701 casos y arroja que las principales manifestaciones iniciales por las cuales los pacientes consultaron son fatiga, artralgia, sinusitis, descarga nasal, tos, hipoacusia, otalgia, fiebre, hiposmia, renales

\begin{tabular}{|c|c|}
\hline Sitio & $\%$ \\
\hline Senos paranasales & 68 \\
\hline Nariz & 51 \\
\hline Oído & 43 \\
\hline Pulmón & 62 \\
\hline Articulaciones & 57 \\
\hline Riñón & 38 \\
\hline Músculo & 30 \\
\hline Ojo & 30 \\
\hline Piel & 27 \\
\hline Traqueo-bronquial & 19 \\
\hline
\end{tabular}

(hematuria, hipertensión, edema), erupción cutánea, oculares y por último nariz en silla de montar, siendo la cabeza y cuello el sitio principal (9).

\section{MANIFESTACIONES RENALES:}

Elcompromiso renal delas vasculitis relacionadas a ANCA es muchas veces expresado como una glomerulonefritis ( $G M N)$ rápidamente progresiva con hematuria, proteinuria, hipertensión o edemas. Esto sin un tratamiento temprano puede avanzar hasta la insuficiencia renal presentándose como una insuficiencia renal aguda, crónica o enfermedad renal terminal, e incluso manifestaciones mortales como la GMN necrotizante $(11,12)$. 


\section{MANIFESTACIONES PULMONARES}

Sus manifestaciones pulmonares son un tanto inespecíficas, se mencionan nódulos, lesiones cavitadas, masas multifocales, pleuritis, tos, sibilancias, derrame pleural, infiltrados pulmonares, y la más temida la hemorragia alveolar difusa que ocasionaría insuficiencia respiratoria potencialmente mortal. El dúo de múltiples nódulos pulmonares cavitados y una obstrucción de la vía aérea pronunciada, son hallazgos importantes que orientan hacia la enfermedad $(11,13)$.

\section{MANIFESTACIONES EN OÍDO}

La mayoría de pacientes con manifestaciones a este nivel tiene una correlación con alteraciones inflamatorias en la mucosa de oído medio y mastoides, inclusive se ha asociado anormalidades a lo largo de la mucosa de la trompa de Eustaquio o en su drenaje, a nivel de la nasofaringe $(5,14,15)$.

Su manifestación más importante es la otitis media crónica serosa, pudiendo asociar una hipoacusia en su mayoría de tipo conductiva, asimismo, pueden encontrarse hipoacusias neurosensoriales y mixtas $(2,15,16)$. Se cree que la afectación neurootológica es causada por efecto compresivo y destructivo de granulomas sobre el nervio vestibulococlear, estas masas afectan el hueso temporal desde la mastoides hacia su porción petrotimpánica.

Algunas hipótesis incluso mencionan el depósito de inmunocomplejos en la cóclea como el causante de la discapacidad auditiva $(15,16,17)$. Otras de las manifestaciones a consecuencia de la afectación otológica son las parálisis del nervio facial, dado a que la porción timpánica de este par craneal atraviesa el oído medio en su porción superior y podría afectarse por compresión o vasculitis en su microvasculatura $(14,15,16)$.

El vértigo ha sido descrito en estos pacientes sin embargo no es algo común, se cree es consecuencia de la afectación vestibular por inmunocomplejos, o siendo este un síntoma del compromiso al sistema nervioso central (17). En el oído externo puede haber lesiones ulcerativas, mismas se han descrito podrían llevar a la estenosis del canal $(16,18)$.

\section{MANIFESTACIONES EN CAVIDADES NASALES}

Laenfermedadnasosinusaleslaexpresión clínica más frecuente de GPA en la cabeza y cuello, los signos y síntomas principales que se presentan son costras en la mucosa, rinosinusitis crónica, obstrucción nasal, descarga serosanguinolenta, cacosmia, hiposmia y epífora por compresión al drenaje del conducto nasolagrimal. $(14,19)$

La vasculitis generalmente ataca primero al plexo de Kiesselbach, siendo el sitio más frecuente de afectación nasosinusal por la GPA, que conforme avanza la enfermedad puede generar una alteración en la arquitectura del tabique septal, que puede culminar en deformidad externa (nariz en silla de montar) $(14,15,16)$. Posteriormente se continúa con su afectación hacia los senos paranasales e incluso podría afectar la base del cráneo $(14,16)$.

En su valoración, la endoscopía es de gran ayuda, sin embargo, la tomografía axial computarizada (TAC) es una herramienta importante en la valoración de la extensión de la enfermedad de manera no invasiva, los signos más importantes encontrados han sido el aumento del grosor de la mucosa, opacificación del seno, erosión 
ósea, neo-osteogénesis, osteítis esclerosante y destrucción ósea $(15,20)$

\section{MANIFESTACIONES ORALES}

Las manifestaciones en cavidad oral son raras, y usualmente se presentan como estomatitis ulcerativa en paladar, mejillas y lengua, además de lesiones eritemato-granulomatosas gingivales con petequias que recuerdan a fresas $(5,18,21,22)$.

\section{MANIFESTACIONES LARÍNGEAS, TRAQUEALES Y FARÍNGEAS}

A este nivel los síntomas más usuales son la disnea, estridor y carraspeo, mientras la tos y disfonía son difíciles de encontrar $(23,24)$. A la visión endoscópica los hallazgos más comunes comprenden úlceras mucosas, edema, eritema y estenosis subglótica, esta última pudiendo presentarse de forma aguda, incluso llegando a requerir una traqueostomía de emergencia (16, 23).

Un estrechamiento de la vía aérea a este nivel lo sufrirá desde un $10-16 \%$ de los pacientes con GPA, asimismo, una disminución del lumen laríngeo a un $75 \%$ podría pasar asintomático, mientras uno del $80 \%$ podría conllevar la muerte si no es tratada, por lo cual es importante descartar patología a este nivel con el fin de resguardar la vía aérea $(16,23,24)$.

\section{MANIFESTACIONES EN OJO Y ÓRBITA}

Esta es una de sus manifestaciones localizadas más temidas ya que podríallevar a una importante morbilidad, lo más común es la afectación a la órbita por una masa granulomatosa que se expandió a través de los senos paranasales hasta el espacio retrorbitario, manifestándose como proptosis, diplopía, fistula nasoorbitaria, edema palpebral e incluso ceguera por compresión al nervio óptico $(5,10,16)$.

Puede afectar el globo ocular ocasionando escleritis y epiescleritis, e incluso pudiendo afectar la vasculatura retiniana (10). En el sistema lacrimal se ha documentado dacrioadenitis, dacriocistoceles y dacriocistitis $(16,25)$.

\section{MANIFESTACIONES EN GLÁNDULAS SALIVALES}

Las manifestaciones en este sitio suelen ser inusuales, y dentro de estas las más afectadas suelen ser las submandibulares y parótida, ocasionando inflamación y dolor, que incluso pueden asemejar abscesos o malignidad (16, 26).

\section{MANIFESTACIONES EN PIEL}

Se observan hasta en la mitad de los casos, su manifestación más común es púrpura en extremidades inferiores. Manifestaciones menos comunes incluyen pápulas y nódulos subcutáneos $(10,27)$.

\section{DIAGNÓSTICO}

En 1990 el Colegio Americano de Reumatología designó 4 criterios diagnósticos para la GPA, estos son: 1) compromiso sinusal, 2) radiografía de tórax con nódulos pulmonares, infiltrado pulmonar fijo o cavidades, 3) urianálisis con glóbulos rojos dismórficos con o sin cilindros hemáticos y 4) granuloma en una arteria o en el espacio perivascular de una arteria o arteriola. Si 2 de estos 4 se cumplen se tiene una sensibilidad del $88,2 \%$ y una especificidad del $92,0 \%$ para el diagnóstico (28). Sin embargo actualmente se

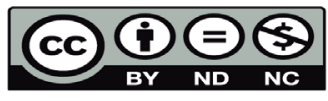


sabe que es un diagnóstico difícil y estos criterios no juegan un rol importante en el diagnóstico como lo hicieron hace unos años, este se basa sobre todo en las manifestaciones clínicas, en asociación con un análisis histopatológico y la positividad del PR3-ANCA, este último correlacionándose de manera directa con la actividad de la enfermedad $(2,10)$.

En la forma localizada de la enfermedad la positividad del ANCA es alrededor del $50 \%$, en comparación del $90 \%$ de la sistémica, por lo tanto el rol de la biopsia en las manifestaciones de cabeza y cuello toman mayor valor, sin embargo, la sensibilidad de este marcador depende de manera directa del estadio y actividad de la patología, y no es estrictamente necesario para su diagnóstico, pudiendo diagnosticarse con síntomas concordantes con un análisis histopatológico $(2,11,29)$.

A pesar de ello, los hallazgos histológicos muchas veces pueden ser inespecíficos en la región de cabeza y cuello, ya que, las dificultades técnicas para obtener los especímenes pueden alterar la calidad de la muestra, resultando en que la triada clásica de vasculitis, necrosis e inflamación granulomatosa se vea tan solo en un $16 \%$. En contraste, la mayoría de biopsias a nivel pulmonar sí las muestran esta triada (29).

En el urianálisis se observan manifestaciones propias de la GMN, glóbulos rojos dismórficos con o sin la presencia de cilindros hemáticos, proteinuria de grado variable y leucocituria, destacando que la $G M N$ es rápidamente progresiva y las pruebas de función renal indicarán el deterioro de su función, con el aumento sostenido de la creatinina sérica.
El análisis de los tejidos renales mostrará una GMN pauci-inmune o necrotizante, y en su presentación aguda podría únicamente presentar necrosis de las paredes vasculares $(11,29,30)$

\section{TRATAMIENTO}

El tratamiento médico inicial se utiliza con el objetivo de inducir la remisión, en otras palabras, la ausencia clínica de actividad de la enfermedad. Existen herramientas para valorar esta como el Birmingham Vasculitis Activity Score (BVAS), que se basa en valores clínicos y de laboratorio $(31,32)$.

Al diagnosticar la enfermedad el tratamiento inicial debe instaurarse rápidamente. Para seleccionar el manejo farmacológico debemos categorizar al paciente en si tiene o no, una lesión que amenace algún órgano o a su vida (estos incluyen, pero no están limitados a: daño renal, hemorragia alveolar de cualquier grado, neuropatía motora, afectación cardiaca, meníngea, retroorbitaria o mesentérica).

Si la presentación inicial de la GPA no amenaza la vida el tratamiento se basa en glucocorticoides (prednisolona $1 \mathrm{mg} / \mathrm{kg} /$ día máximo $80 \mathrm{mg} /$ díaoral) asociados a metrotexate (20-25 mg/semana, oral o parenteral) o micofenolato de Mofetil (hasta $3 \mathrm{~g} /$ día oral), la dosis de prednisolona se debe de disminuir gradualmente, y así tener una meta de 7,5-10 $\mathrm{mg} /$ día para el mes 3 de tratamiento, sin embargo en la práctica este objetivo puede tomar hasta 5 meses $(31,32,33)$.

Si al momento instaurar el tratamiento inicial hay datos de amenaza a algún órgano o la vida del paciente, o si se tratase de una recaída grave se 
recomienda glucocorticoides con ciclofosfamida (en pulsos intravenosos inicialmente cada 2 semanas e ir aumentando a cada 3 semanas, la dosis recomendada de la Sociedad Británica de Reumatología de $15 \mathrm{mg} / \mathrm{kg}$ ), o rituximab (375 $\mathrm{mg} / \mathrm{m} 2 /$ semana intravenoso por 4 semanas).

Es importante recordar que la la toxicidad de la ciclofosfamida a largo plazo depende de sus dosis acumulativas, la dosis máxima en toda la vida no debería ser mayor de 25 gramos para evitarla. Si en la presentación inicial se obtiene un valor de creatinina sérica mayor a $5,7 \mathrm{mg} / \mathrm{dL}$ o hay una hemorragia alveolar se recomienda el uso de plasmaféresis, ya que se ha demostrado que esta previene la enfermedad renal terminal o muerte a 3 meses $(31,32,33,34)$

Una vez conseguida la remisión se recomienda un tratamiento de mantenimiento con glucocorticoides a dosis bajas junto con metrotexate (20-25 mg/semana, oral o parenteral), azatriopina (2 $\mathrm{mg} / \mathrm{kd} / \mathrm{día}$ oral), rituximab (1 gramo cada 4-6 meses intravenoso) o micofenolato de mofetil ( $2 \mathrm{~g} /$ día oral). Se debe mantener por 24 meses y posterior de este tiempo regular las dosis a la baja para intentar una remisión sin medicamentos, sin embargo las recaídas son comunes $(31,32,33)$

Los efectos adversos de estos tratamientos son amplios e inclusive podrían conducir a la muerte, dentro de sus consecuencias más frecuentes se encuentran las infecciones, ya que estos encuentran un medio inmunocomprometido por efecto farmacológico y pueden complicar rápidamente $(10,16,31)$.

El tratamiento quirúrgico es limitado, sin embargo, se recomienda como una alternativa solamente cuando el tratamiento sistémico ha fallado, ya que en estos pacientes hay un importante problema en la perfusión del tejido afectado y un procedimiento quirúrgico puede empeorar la clínica del paciente o no tener los resultados deseados.

El impacto en la calidad vida del paciente de algunos síntomas, o el impacto psicológico de las deformidades nasales llevan a que las correcciones quirúrgicas sean necesarias en algunos casos $(14,16)$

A nivel laringo-traqueal la consolidación de una vía aérea es fundamental, solo 1 de cada 5 pacientes necesitará procedimientos a este nivel, dentro de los que se mencionan la dilatación endoscópica, la resección del segmento estenótico, y la escisión con láser. La traqueostomía se reserva para pacientes severamente afectados $(14,16,24)$.

\section{CONCLUSIONES}

La GPA es una enfermedad poco común, con un curso natural severo, y cuyas manifestaciones iniciales van a encontrarse principalmente en la cabeza y el cuello, siendo el otorrinolaringólogo uno de los primeros médicos que va a tener contacto con estos pacientes. Por lo tanto, el conocer estas manifestaciones, el diagnóstico de la enfermedad y su manejo, es de suma importancia para disminuir la morbimortalidad de la misma y sus consecuencias irreversibles, con el fin de mejorar la calidad de vida del paciente y su pronóstico.

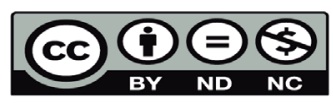




\section{REFERENCIAS BILBIOGRÁFICAS}

1. Jennette JC, Falk RJ, Hussein Gasim A. Pathogenesis of ANCA vasculitis. Current Opinion in Nephrology and Hypertension. 2011May;20(3):263-70.

2. Schilder AM. Wegener's Granulomatosis vasculitis and granuloma. Autoimmunity Reviews. 2010;9:483-7.

3. Ntatsaki E, Watts RA, Scott DGI. Epidemiology of ANCA-associated Vasculitis. Rheumatic disease clinics. 2010Aug;36(3):447-61.

4. Katsuyama T, Sada K-E, Makino H. Current concept and epidemiology of systemic Vasculitides. Allergology international. 2014Dec;63(4):505-13.

5. Holle JU, Laudien M, Gross WL. Clinical manifestations and treatment of Wegener's Granulomatosis. Rheumatic Disease Clinics. 2010Aug;36(3):507-26.

6. Gomez-Puerta JA, Bosch X. Anti-neutrophil cytoplasmic Antibody Pathogenesis in small- vessel Vasculitis. The American Journal of Pathology. 2009Nov5;175(5):1790-8.

7. Hoffman G, Kerr GS, Leavit RY, Hallahan CW. Wegener granulomatosis: an analysis of 158 patients. Annals of Internal Medicine. 1992Mar15;116(6):488-98.

8. Taylor SC, Clayburgh DR, Rosenbaum JT, Schindler JS. Pregression and manegement of Wegener's hgranuloomatosis in the head and neck. The Laryngoscope. 2012Aug;122:1695700 .

9. Abdou NI, Kullman GJ, Hoffman GF, Sharp GC, Specks U, McDonald T, et al. Wegener's Granulomatosis: Survey of 701 Patients in North America. Changes in Outcome in the 1990s. The Journal of Rheumathology. 2002;29(2):309-16.

10. Kuabaisi B, Samra KA, Foster CS. Granulomatosis with polyangiitis (Wegener's disease): An updated review of ocular disease manifestations. Intractable \& Rare Diseases Research. 2016May;5(2):61-9.

11. Greco A, Marinelli C, Fusconi M, Macri GF, Gallo A, De Virgilio A, et al. Clinic manifestations in granulomatosis with polyangiitis. International Journal of Immunopathology \&amp; Pharmacology. 2016Jun;29(2):1-7.

12. DeGroot K. Renal disease in small-vessel vasculitis. Cleveland Clinic Journal of Medicine. 2012Nov;79(2):22-6.

13. Gildea TR. Pulmonary disease in small-vessel vasculitis. Cleveland Clinic Journal Of Medicine. 2012Nov;79(3):27-30. 
14. Alam DM, Seth $R$, Sindwani $R$, Woodson EA, Rajasekaran K. Upper airway manifestations of granulomatosis with polyangiitis. Cleveland Clinic Journal of Medicine. 2012Nov;79(3):S16S21.

15. Sharma A, Deshmukh S, Dabholkar J. ENT manifestations of Wegeners Granulomatosis. Otolaryngologia Polska. 2013;67:257-60.

16. Wojciechowska J, Krajewski W, Krajewski P, Krecicki T. Granulomatosis With Polyangeiitis in Otolaryngologist Practice: a review of Current Knowledge. Clinical and experimental Otolaryngology. 2016Mar;9(1):8-13.

17. Takagi D, Nakamaru Y, Maguchi S, Furuta Y, Fukuda S. Otologic manifestations of Wegener's granulomatosis. The Laryngoscope. 2002Sep;112:1684-90.

18. McCaffret TV, McDonald TJ, Facer GW, DeRemee RA. Otologic manifestations of Wegener's granulomatosis. Otolaryngology-Head and Neck Surgery. 1980Sep1;88(5):586-93.

19. Cannady SS, Batra PS, Koening C, Lorenz RR, Citardi MJ, Langford C, et al. Sinonasal Wegener Granulomatosis: A Single-Institution Experience With 120 Cases. The Laryngoscope. 2009Apr;119:757-61.

20. Grindler D, Cannady S, Batra PS. Computed tomography findings in sinonasal Wegener's granulomatosis. American Journal of Rhinology \& Allergy. 2009Sep;23(5):497-501.

21. Candau A, Valenzuela B, Dean A, Alamillos FJ. Wegener's Granulomatosis With Oral Mucosal Involvement as First Manifestation. Acta Otorrinolaringológica Española. 2014;65(1):53-5.

22. Stewart C, Cohen D, Bhattacharyya I, Scheitler L, Riley S, Calamia K, et al. Oral manifestations of Wegener's granulomatosis A report of three cases and a literature review. The Journal of the American Dental Association . 2007Mar;138(3):338-48.

23. Vega Braga FL, Machado de Carvalho G, Caixeta Guimarães A, Scaramussa L, ordão Gusmão R. Manifestaciones otorrinolaringológicas de la granulomatosis de Wegener. Acta Otorrinolaringológica Española. 2013;64(1):45-9.

24. Solans-Laqué R, Bosch-Gil JA, Canela M, Lorente J, Pallisa E, Vilardell-Tarrés M. Clinical features and therapeutic management of subglottic stenosis in patients with Wegener's granulomatosis. Lupus. 2008Sep;17(9):832-6.

25. Westbrook BJ, Scurry WC, Hudak DT, McGinn J, Stack Jr BC. Recurrent bilateral dacryocystoceles in Wegeners granulomatosis: a rhinologic perspective. American Journal of Otolaryngology-Head and neck medicine. 2007;27:409-12.

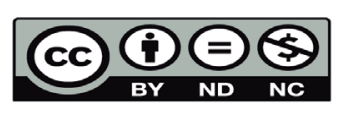


26. Gassling V, Wiltfang Jörg, Hampe J, Bräsen JH, Both M, Moosig F. Salivary Gland Swelling In Wegener's Granulomatosis: A Rare Cause Of A Frequent Symptom. The Journal Of Rheumatology . 2010Dec;37(12):2633-5.

27. Comfere NI, Macaron NC, Gibson LE. Cutaneous manifestations of Wegener's granulomatosis a clinicopathologic study of 17 patients and correlation to antineutrophil cytoplasmic antibody status. Journal of Cutaneous Pathology. 20070ct;34(10):739-47.

28. Leavitt RY, Fauci AS, Bloch DA, Michel BA, Hunder GG, Arend WP, et al. The American College Of Rheumatology 1990 Criteria For The Classification Of Wegener's Granulomatosis. Arthritis and Rheumatology. 1990Aug;33(8):1101-7.

29. Jeannette JC, Nachman PH. ANCA Glomerulonephritis and Vasculitis. Clinical Journal of American Society of Nephrology. 20160ct;12(10).

30. Grygiel-Górniak B, Limphaibool N, Perkowska K, Puszczewicz M. Clinical manifestations of granulomatosis with polyangiitis: key considerations and major features. Postgraduate medicine. 2018Jan;:581-96.

31. Yates M, Watts RA, Bajema IM, Cid MC, Crestani B, Hellmich B, et al. EULAR/ERA-EDTA recommendations for the management of ANCA-associated vasculitis . Annals of the Rheumatic Diseases. 2017Aug1;75(9):1583-94.

32. Ntatsaki E, Carruthers D, Chakravarty K, D'Cruz D, Harper L, Jayne D, et al. BSR and BHPR guideline for the management of adults with ANCA-associated vasculitis. Rheumatology. 2014Dec;53(12):2306-9.

33. Lynch III JP, Derhovanessian A, Tazelaar H, Belperio JA. Granulomatosis with Polyangiitis (Wegener's Granulomatosis): Evolving Concepts in Treatment. Seminars in Respiratory and Critical Care Medici. 2018;39:434-58.

34. Yates M, Watts R. ANCA-associated vasculitis. Royal College of Physicians, Clinical Medicine. 2017Feb;17(1):60-4. 